\title{
The Dynamics of EU-Africa Research and Innovation Cooperation Programmes
}

\author{
Erika Kraemer-Mbula, Constantine Vaitsas, \\ and George Owusu Essegbey
}

\begin{abstract}
This chapter focuses on the practical achievements of existing Africa-Europe science, technology and innovation (STI) projects. It reviews six programmes that fund Africa-Europe STI cooperation, highlighting some of their successful cooperative projects, particularly in the fields of new water and sanitation technologies and green chemicals. This practical focus sheds light on the intrinsically unequal cooperation patterns among African countries. Participation of a diverse range of African partners, and private sector participation in Africa-EU STI cooperation, remain limited. The authors thus point out that future cooperation should focus on how to market and disseminate STI products and services.
\end{abstract}

E. Kraemer-Mbula $(\bowtie)$

University of Johannesburg, Johannesburg, South Africa

C. Vaitsas

Forth/Praxi Network, Athens, Greece

G.O. Essegbey

Science and Technology Policy Research Institute of the Council for Scientific and Industrial Research, Accra, Ghana

(C) The Author(s) 2018

A. Cherry et al. (eds.), Africa-Europe Research and Innovation

Cooperation, https://doi.org/10.1007/978-3-319-69929-5_3 
Keywords Framework programme • Horizon 2020 • European Union - African Union • Balanced cooperation $\bullet$ Co-financing $\bullet$ Private sector

- Business networks

\section{INTRODUCTION}

The landscape of Africa-Europe collaboration in science, technology and innovation (STI) is becoming increasingly complex. Thematic areas are now addressing global, multi-sectoral concerns such as climate change; the conventional principles of "donorship" are being replaced by a growing search for equal partners and co-funding; and the need to address the global scientific divide and strengthen the STI capacities of low- and middle-income economies is more and more widely acknowledged. How have African countries and organisations, in particular, been affected by or contributed to these changes?

To answer this question, this chapter reviews past, present and future collaboration patterns of six funding programmes in which African and European countries and organisations have participated. These programmes are the European Commission's (EC) Framework Programmes (FP), with particular emphasis on the last two iterations, FP7 and Horizon 2020; the Cooperation in Science and Technology (COST) programme; Eurostars; the African, Caribbean and Pacific (ACP) countries' Science and Technology (S\&T) programme; the European \& Developing Countries Clinical Trials Partnership (EDCTP); and the African Union Research Grants (AURG) programme. Most of these programmes, with the exception of the AURG, are set in the context of the European Union (EU). As such, they should enable African partners to become important players in a bi-regional cooperation context. The chapter first highlights outstanding examples of successful and innovative projects funded under these programmes and then reflects on the way future collaborative relations can be strengthened.

\section{The Seventh Framework Programme}

The EU's first FP for research was introduced in 1984 to boost the scientific and economic development of the European Community, while promoting international cooperation. Its seventh iteration (FP7) was 
Start date: 2007 - End date: 2013

Total budget: 50 billion euros

Funding body: European Union

In brief: FP7 was the key tool for the EU to respond to its needs in terms of employment and competitiveness. Its main objectives were to strengthen the scientific and technologic base of the European industry and to encourage international competitiveness.

Fig. 3.1 Overview of the Seventh Framework Programme (FP7)

launched in 2007. One of FP7's guiding ideas was that the promotion of the EU's strategic goals on research and development (R\&D) was to be achieved through facilitated partnerships with third countries (i.e. countries outside of the EU, thus including African states) while addressing specific challenges that third countries face or which have a global impact (e.g. climate change). The programme had five major building blocks (see also Fig. 3.1):

- Cooperation: fostering collaborative research across Europe and other partner countries in different thematic areas such as health, food, agriculture and fisheries, nanoscience, environment and transport

- Ideas: supporting research on the basis of scientific excellence in areas including engineering, socio-economic sciences and the humanities

- People: supporting research mobility and career development both for the EU and internationally

- Capacities: strengthening the research capacities for the EU regarding research infrastructure, research potential, science in society and specific activities of international cooperation

- Nuclear Research: including research, technological development, international cooperation, dissemination of technical information and exploitation activities

For the first time, FP7 provided a broad opening for international cooperation in programmes and research schemes across the entire FP, while setting collaboration priorities with third countries and regions 
across the thematic work programmes. By defining specific actions for collaboration with third countries and regions in each of the thematic programmes, FP7 ensured that budgets for international cooperation were included at the level of each relevant call for proposals. Finally, the principle of partnership and dialogue was intensively applied in the specific actions for international cooperation with third countries and regions, in particular through the so-called INCO-NET instrument, of which CAAST-Net and CAAST-Net Plus are examples.

African participation in the FP7 rose dramatically. As is also reported in Chap. 2, 1315 participants from organisations in 45 African countries took part in 565 EU-funded projects, with a total budget of 178 million euros. In comparison, FP6 counted, in 2006, 882 African participants for 322 research projects, for an allocated budget of 95 million euros from the EU. ${ }^{1}$ Under FP7, and as Fig. 3.3 also shows, South Africa, followed by Ghana, Uganda and Kenya, was the leading partner in terms of project participation.

Universities and governmental research institutions have been, so far, the leading project participants. In South Africa, the universities of Cape Town, KwaZulu-Natal and Pretoria, along with the Council for Scientific and Industrial Research, the Agricultural Research Council, the Institute of Natural Research Association and the National Research Foundation, were frequent FP7 participants. In Kenya, the University of Nairobi, the Ministry of Education, Science and Technology and the International Centre for Research in Agroforestry have been particularly active. Although fewer Ghanaian research organisations and universities participated in the programmes, the University of Ghana, the Kwame Nkrumah University of Science and Technology Kumasi and the Council for Scientific and Industrial Research were among the most active members. While some South African small- and medium-sized enterprises (SMEs) were involved, such as Research Africa, SME participation has remained limited to date, a trend that applies to most African countries (Fig. 3.2).

African FP7 participation received a new impetus with the 2010 Coordinated Call for Africa, also known as the "Africa Call", which placed African needs and priority research areas at the centre of the funding and programme design. It launched with the aim of addressing some of the S\&T objectives of the Joint Africa-EU Strategy (JAES), in particular those of the Eighth Partnership on Science, Information Society and Space, 


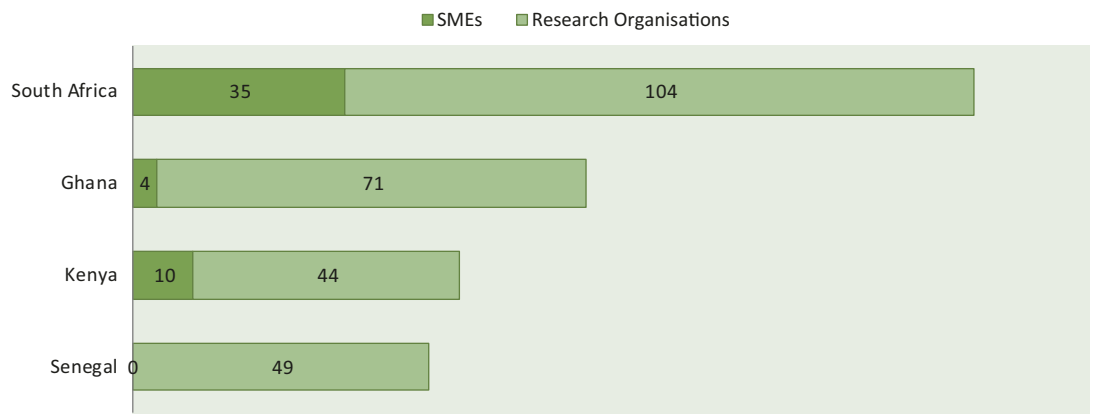

Fig. 3.2 SMEs and research organisations' participation in FP7 (Source: eCorda 2015)

seeking more particularly the joint elaboration of the Partnership's lighthouse projects by the African Union Commission (AUC) and the EC. The call funded 26 projects in three thematic areas: 15 on health, 7 on environment (including climate change), and 4 on food, agriculture fisheries and biotechnology. The funded projects intended to move away from a "donorship" approach to more equal partnership terms, combining the latest scientific discoveries with local knowledge to maximise research impact. Projects also aimed to strengthen local capacities in the relevant S\&T fields and their applications, through training activities and the exchange of staff.

Again, South Africa was the most active country in the Africa Call, participating in 12 projects. In fact, South Africa's success under the FP7 was largely a result of a concerted effort to promote cooperation, undertaken by the European South African Science and Technology Advancement programme, an advisory, information and support platform for researchers, funded under FP7 and implemented by the South African Department of Science and Technology, as well as South Africa's FP7 Network of National Contact Points (NCPs) (EC 2009). As Fig. 3.3 shows, South Africa was followed by Tanzania (with 11 projects), Uganda (10) and Burkina Faso (10).

Within this context, the Water, Sanitation and Hygiene Technologies (WASHTech) project stands out for its contribution to the transfer of scientific knowledge through open access. It was a three-year action 
44 E. KRAEMER-MBULA ET AL.




research initiative, spanning 2011-2013, backed by more than 2 million euros in funding, and involving African partners from Burkina Faso, Ghana and Uganda. The project was based on the premise that although the water and sanitation sectors are undergoing many changes, new technologies developed in these fields are simply not included in national strategies. The project resulted in two specific outputs: first, the development and introduction of an assessment tool, the Technology Applicability Framework, which provides a neutral approach for investigation of water, sanitation and hygiene (WASH) technological innovation; and second, the establishment of the Technology Introduction Process, which are multi-stakeholder and country-specific guidelines with agreed responsibilities to the successful introduction and uptake of WASH technologies in areas promising sustainable WASH service delivery. The project was set up to establish the required capacities, in order for these technologies to be incorporated in national policies. Moreover, WASHTech differentiated itself from other FP7 projects under the Africa Call by opening its outputs to validate water and sanitation technologies to the public domain. As such, it is an interesting case of non-commercial innovation, currently used in Europe and Africa and with potentially far-reaching impacts in both Europe and Africa.

Although FP7 offered an opportunity for African countries to collaborate with their European counterparts, the private sector showed limited participation. Considered to be the motor of innovation and technology diffusion in Africa's market-based economies, the private sector has the potential to bring co-funding opportunities in research cooperation and strengthen the sustainability of research networks. Future developments within bi-regional scientific collaborations have specifically sought to take this issue into consideration.

\section{Horizon 2020}

Succeeding FP7 in 2014, Horizon 2020 is the largest EU research and innovation (R\&I) programme to date and is also expected to attract additional private investment funds based on the estimated results generated when entering the market. It has three pillars (Fig. 3.4): 
Start date: 2014 - End date: 2020

Total budget: 80 billion euros

Funding body: European Commission

In brief: Horizon 2020 is the biggest EU Research and Innovation programme ever. Its main objective is to ensure Europe produces world-class science focusing on excellent science, industrial leadership and tackling societal challenges.

Fig. 3.4 Overview of Horizon 2020

- Excellence of science to reinforce and extend the EU science base

- Industrial leadership focusing on speeding up the R\&D process behind new technologies and innovations that enable SMEs to grow

- Societal challenges reflecting the policy priorities of the EU strategy for 2020 and addressing major concerns by citizens in the EU and elsewhere (such as health and demographic change, food security, clean energy, transport, climate change and security)

Horizon 2020 is particularly geared towards acquiring additional financing through increasing the number of topics explicitly flagging "international collaboration", which increased from 12\% of topics in FP7 to over $27 \%$ in the $2014-2017$ round of funding calls. It also facilitates worldwide participation by reducing bureaucracy so that participants can focus on the substance of their R\&I endeavours. Despite these efforts, the share of participation of partners from third countries in grant agreements for collaborative actions has fallen from $4.9 \%$ in the FP7 to $2.4 \%$ under Horizon 2020. As of October 2016, AU entities had 191 participations in 79 signed grants, receiving 31.2 million euros from the EU, while 2.9 million euros is from the non-EU budget. The equivalent first two years of FP7, however, counted with a larger African participation with 368 partners from 37 African countries involved.

As Figs. 3.5 and 3.6 show, South Africa still leads African participation in Horizon 2020 projects, as it did under FP7, followed by Ghana, Kenya and Uganda. The participation of the SMEs has remained, once again, limited. 


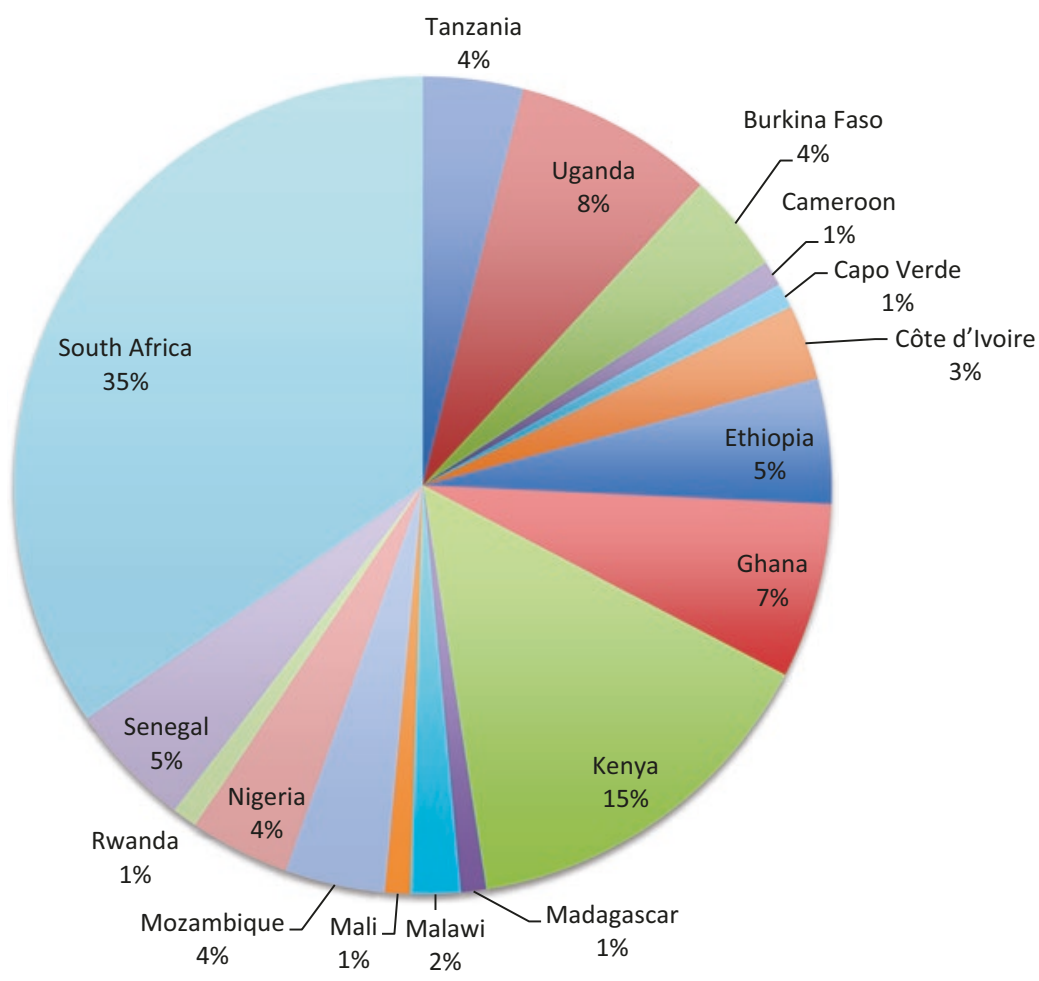

Fig. 3.5 Sub-Saharan African participation in Horizon 2020 (Source: eCorda, May 2016)

Most Sub-Saharan African organisations that answered the specific programme calls are research centres, institutions, universities and ministries. In the cases of South Africa, Ghana and Kenya, the most frequently participating organisations are the same noted in the case of FP7. In the case of Uganda, Makerere University is the leading participant, having joined already five different ongoing projects. Other frequent participants include the Uganda National Health Research Organisation and the National Agricultural Research Organisation. 


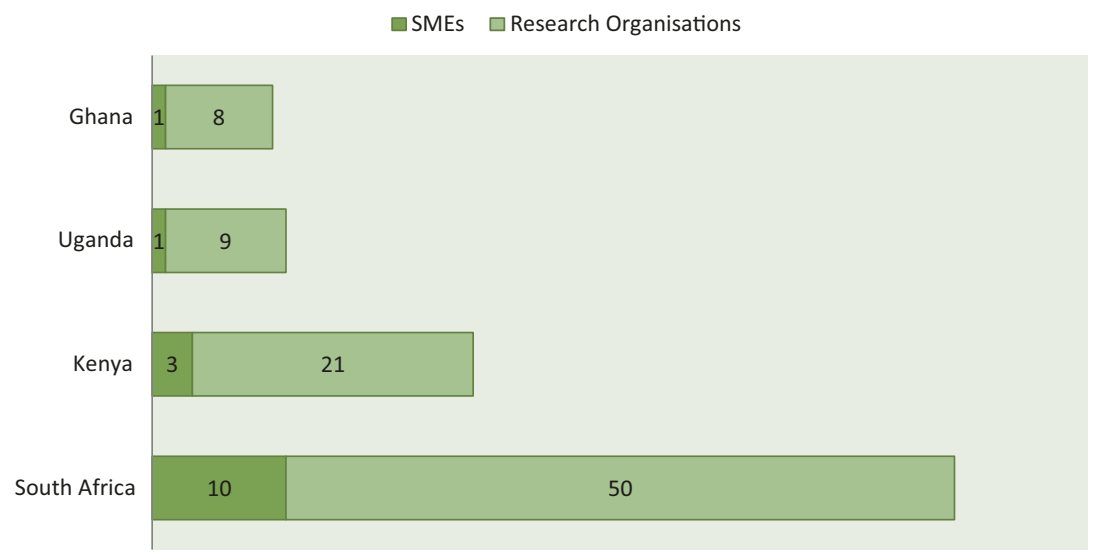

Fig. 3.6 SME and research organisations' participation in Horizon 2020 (Source: eCorda, May 2016; ECorda is the database of the "Common Research Datawarehouse, collecting proposals, evaluation and grant management data of all the operational systems automating key business processes around The Framework Programmes, H2020 - FP5" (Source: European Commission, "eCORDA and Cordis", November 2016, Available from http://www.ncpacademy.eu/wp-content/uploads/2016/ 11/20161103_eCORDA-and-CORDIS.pdf. Accessed 12 December 2017).)

Outcome Testimonial: Horizon 2020 information sessions for building biregional partnerships. Compiled by Emeka Orji (National Office for Technology Acquisition and Promotion, Nigeria) and Melissa Plath (Finnish University Partnership for International Development, University of Jyväskylä).

Access to information on research funding opportunities, particularly those from the EU, has often been difficult for researchers outside of Europe. EU funding can be a key instrument for supporting the formation and development of long-term, bi-regional research partnerships. To help address this asymmetry, CAAST-Net Plus organised a series of activities to disseminate information on opportunities for African participation in Horizon 2020, the EU's programme for funding research and innovation (2014-2020). For example, a Horizon 2020 workshop was held in Nigeria in 2013 to foster the participation of a wide spectrum of researchers.

Between 2013-2016, CAAST-Net Plus organised 15 workshops and information sessions on Horizon 2020, in 8 different African countries, with over 1100 participants from 28 different African countries. The events provided information on the relevant calls, rules, and strategies for successful proposals to Horizon 2020. Beyond providing information to the scientific community, CAAST-Net Plus also supported the nomination and training 
of an estimated 100 nominated or likely National Contact Points (NCPS) in Africa. NCPs are key disseminators of information on Horizon 2020: they can provide tailored advice and support to national researchers. CAASTNet Plus organised five trainings for NCPs, inviting nominated or likely NCPs from across Africa.

As a result of these activities, and taking Nigeria as an example, researchers became more aware of the opportunities offered by Horizon 2020 and the potential of bi-regional partnerships in developing research proposals. Numerous Nigerian researchers requested to participate in various projects. While it is too early to observe specific or measurement outcomes from this work, we do know that many of those who have attended the Horizon 2020 information sessions are actively engaged in proposals for bi-regional research grants, which is likely to result in a higher degree of African participation research and innovation projects, many under African leadership.

In CAAST-Net Plus, we have experienced first-hand how more equal access to information and opportunities is important for building more-and more equal-bi-regional partnerships. Within our own partnership, we have noticed that when partners have equal access to information, they are more willing and able to contribute. We have also seen that supporting the nomination and training of NCPs contributes to knowledge sharing within a country. As disseminators of information, they play a key role in ensuring more equal access to information. CAAST-Net Plus has thus delivered a valuable contribution to the development of bi-regional research partnerships.

Since most projects funded through Horizon 2020 are still ongoing, it is premature to discuss the impact of Horizon 2020 on bi-regional cooperation. Instead, we introduce two current projects that reflect the type of programming work undertaken in Horizon 2020.

- Africa-EU innovation alliance for water and climate (AfriAlliance). The AfriAlliance project (2016-2021) is designed to foster collaboration between European and African actors in the fields of water innovation, research, policy and capacity development with a strategic objective to prepare African countries for future climate challenges. Although several initiatives and networks are active in this field, they remain fragmented. This project endeavours to connect and consolidate them and establish an overall coordination platform. The project has been allocated more than 3 million euros. Participation from Sub-Saharan Africa is significant, and includes partners from South Africa, Cote d'Ivoire, Ghana and Burkina Faso. 
- Integrated aquaculture based on sustainable water recirculating system for the Victoria Lake Basin (VicInAqua). The VicInAqua project (2016-2019) aims at developing sustainable sanitation and recirculating aquaculture systems for the wastewater treatment and its reuse in agriculture in the Victoria Lake basin. The project tackles issues of food and health security while contributing to the protection of the ecosystems in Lake Victoria. VicInAqua is fully funded by the EC under Horizon 2020 with almost 3 million euros. Together with European participants, research associations and ministries from Uganda and Kenya will contribute to the development of a novel self-cleaning filter, which will be used in the aforementioned area.

\section{Cooperation in Science and Technology (COST)}

The European Economic Community and 19 European countries established the COST programme in 1971 to promote networks of researchers throughout Europe and beyond. Today, COST consists of 36 member countries, one cooperating state (Israel) and several international partner countries. Its objectives include capacity building through connecting high-quality scientific groups, offering networking opportunities for early stage researchers and increasing the impact of the current research among policymakers, regulatory bodies and the private sector (Fig. 3.7).

Although the programme does not fund research itself, it supports the creation of bottom-up networks of scientists and researchers, through the

Start date: 1971 - present

Annual budget per COST Action: average of 130.000 euros per Action

Funding body: European Commission and national budgets

In brief: COST is an intergovernmental organisation promoting the cooperation on science and technology research through creating networks, also known as "COST Actions". Those networks allow scientists to share their ideas with their peers, leading to proper dissemination of research and innovation inside and outside EU borders.

Fig. 3.7 Overview of the Cooperation in Science and Technology programme 
so-called COST Actions. These are essentially networking tools to promote international coordination of nationally funded research and global cooperation. Typically, research must be relevant for at least five COST countries, while the financial support totals 130,000 euros per year for a four-year period-and must encourage global cooperation. The African countries already participating in COST Actions include Ethiopia, Mauritius, Namibia, South Africa and Sudan. Green chemistry, presented below, is a meaningful example of successful COST collaboration outside of the EU.

- Cooperation between scientists from the United Kingdom and Ethiopia in the field of green chemistry (COST 2017a). Green chemistry focuses on the design and use of less hazardous chemicals and processes. It has become particularly relevant in African countries, such as Ethiopia, as it offers unique opportunities to discover new biologically active molecules (for use in pharmaceuticals or agrochemicals) from the wide variety of flora endemic to the region. The collaboration of British and Ethiopian scientists produced a report comparing different methods for oil extraction in Ethiopia, researching milder chemicals for the extraction process. The findings were presented in major "green chemistry" conference organised in Germany in October 2004, and where the researchers from Ethiopia joined the COST Action D29 (COST 2017b). Through the COST Action, scientists were able to raise awareness about the field of green chemistry and to achieve a meaningful and efficient collaboration between the United Kingdom and Ethiopia. The project was considered a success: the report was widely acknowledged and adopted as teaching material from the New University of Lisbon (Engida et al. 2007). Most importantly, it gave visibility to indigenous chemical processes in Ethiopia that match the majority of the principles of green chemistry.

\section{Eurostars}

Together with EUREKA, an intergovernmental organisation for panEuropean R\&D funding and coordination, the EC established the Eurostars joint programme in 2008 (Fig. 3.8) (EUREKA 2017) to 
Start date: 2008 - present Total budget: 1.4 billion euros Funding body: EU (861 million euros) and national funding (287 million euros) In brief: Eurostars is a joint programme between EUREKA and the European Commission, which supports international innovative projects in order to promote the development of innovative products, processes and services.

Fig. 3.8 Overview of the EUREKA programme

support international innovative projects led by R\&D-driven SMEs, with the objective of bringing innovative products, processes and services to European and international markets. As it focuses on innovative, rapidly marketable products, processes and services, the selection process is highly competitive-there is no restriction on the technological area targeted; the only requirement is the clear aim to develop a new product, process or service. Any type of organisation can join a project consortium provided that the main partner is an R\&D-driven SME.

While there was no African participation in the first Eurostars (2008-2014), its second iteration (2014-2020) saw South Africa become the only African country participating in EUREKA, signing in March 2016. Given its recent participation, the country is not yet active in any project under Eurostars calls. South Africa is nonetheless involved in two projects under ACQUEAU (EUREKA's cluster for water), described below.

- The mine water as a resource (MINWARE) project (2014-2017) aims to develop and demonstrate environmentally friendly solutions for mine wastewater from the mining and metal industries. The focus is on developing solutions for acidic metal-laden wastewaters in order to recover the valuable metals with new and less invasive methods and processes, together with the creation of viable economic solutions that are applicable worldwide. The project runs on a budget of 3 million euros, distributed within a consortium including research organisations, large technology providers, SMEs and their end users from Finland, South Africa and Sweden. South Africa's role is to foster the involvement of South African SMEs as end users, specialised in designing and developing biological methods for wastewater treatment. 
- A long-term, sustainable treatment option for acid mine drainage (AMD), the VitaSOFT project, implemented over a 30-month period, focused on the demonstration of a treatment process for AMD. Under the coordination of Vitaone8, a South African SME specialised in developing innovative water treatment technologies, and participants from the United Kingdom and South Africa focused on the successful demonstration of the VitaSOFT process. VitaSOFT is an active biological sulphate-reducing process, designed to reduce the volume of solid waste and provides the potential to recover valuable by-products. The process can also be used for the disposal of other waste such as industrial biodegradable organic waste, which would reduce the cost and the risk that companies take to dispose of waste themselves. The project was financed under the ACQUEAU RTD project call, with a 1 million euros grant co-funded by the EU, South Africa and the United Kingdom.

\section{African, Caribbean and Pacific Countries' Science and Technology (S\&T) Programme}

Funded by the EU and implemented by the ACP secretariat, the ACP S\&T programme responds to the need for joint and systemic approaches in support of STI. The programme acknowledges a direct link between building and enhancing strong S\&T capacities to support research, development and innovation in the ACP region, and the identification and formulation of activities, processes and policies critical to sustainable development. Its first iteration, launched in 2008, funded 36 projects (out of 200 proposals), ranging from quality health care, environmental research activities, energy, transport, agriculture and agro-industry as well as sustainable trade, which received a total of 58 million euros. Its second iteration, launched in 2013 , funded 21 projects focusing more specifically on energy and agriculture with a total budget of 20.8 million euros (Fig. 3.9).

These successive ACP S\&T programmes aim at reducing the scientific and technological divide between ACP countries and the most industrialised countries while increasing partners' ability to better assess their research needs, build stronger networks and implement research politics; strengthening the STI capacities for ACP countries to create, update and 
Starting date: 2008 - present

Total budget: 78 million euros

Funding body: European Union

In brief: ACP SET Programme is a cooperation programme between the EU and the ACP countries, focusing on the enhancement of the internal capacity in SET of ACP countries to support research, development and innovation in their regions.

Fig. 3.9 Overview of the African, Caribbean and Pacific countries' science and technology programme

use scientific knowledge; and enhancing the use of STI as a key enabler for poverty reduction, growth and socio-economic development. The funded projects are expected to establish or strengthen networks between ACP countries, but also to create global links. To successfully address its goals, the programme is designed to support the efforts of ACP countries on three levels (1) institutional, administrative and policy-making; (2) academic, research and technology; and (3) business and civil society. Two successful examples of such projects supported under the ACP S\&T programmes are described below.

- Western Africa biowastes for energy and fertiliser (WABEF) is a research-development and capacity building project focused on the most effective ways to recycle organic residues and produce energy and fertilisers in West Africa. Waste management is a priority in the region, mainly due to the rapid population growth and high urbanisation rates. Therefore, this initiative supported the development of practices and technologies that prioritise both the recycling but also the repurposing of organic waste in the region. Using existing knowledge, the project aimed to devise tools in a participatory way, involving executives and public decision makers, NGOs and teachers, municipalities and agribusinesses, in order to assess the process of anaerobic digestion as the most applicable process for West Africa's waste treatment policy. The project was launched in 2014 for three years, receiving more than 700,000 euros from the EU. Participation from African countries included organisations from Senegal, Benin and Mali. 
- Strengthening capacities and informing policies for developing value chains of neglected and underutilised crops in Africa. This project focused on the research into neglected or underused species and the development of national action plans in order to incorporate them in agricultural systems in Eastern, Western and Southern Africa. Neglected or underused plant species offer niche markets and incomes for poor farmers, as well as options for climate change adaptation. However, their potential is often neglected, mainly due to globalisation, population growth and urbanisation. The project aimed at changing this situation, by involving local stakeholders such as farmers, processors, researchers and the private sector in innovation platforms to upgrade the value chains of such crops. The project ran from 2014 to 2017 and received almost 1 million euros from the EU. Participation from African countries included research organisations and universities in Kenya, Benin and Zimbabwe.

\section{European \& Developing Countries Clinical Trials PARTNERSHIP (EDCTP)}

The EDCTP programme was launched in 2003 as a European response to the need to enhance research collaborations between scientists and accelerate the clinical development for new or improved solutions to tackle poverty-related or neglected diseases such as HIV/AIDS, tuberculosis and malaria (see also Chap. 6). All projects funded under the EDCTP are implemented by partnerships between African and European research institutions in collaboration with the pharmaceutical industry. The EDCTP Association has members from 14 African countries (namely Burkina Faso, Cameroon, Congo, Gabon, The Gambia, Ghana, Mali, Mozambique, Niger, Senegal, South Africa, Tanzania, Uganda and Zambia) and 14 European countries (Austria, Denmark, Finland, France, Germany, Ireland, Italy, Luxembourg, Netherlands, Norway, Portugal, Spain, Sweden and the United Kingdom); it also remains opened to new memberships (Fig. 3.10).

The first EDCTP (2003-2015) received 378 million euros from the EU and European countries that are members of the EDCTP Association. It funded 246 projects for a total cost of 212 million euros (see EDCTP $2014)$. The second programme (2014-2024) received 700 million 
Start date: 2003 - End date: 2024

Total budget: 378 million euros (for the $1^{\text {st }}$ programme) 683 million euros (for the $2^{\text {nd }}$ programme)

Funding body: European Union and European member states

In brief: EDCTP is a public-private partnership between the EU, Sub-Saharan Africa countries and European countries, which focuses on the development of new or improved. interventions for the prevention or treatment of several diseases in Sub-Saharan Africa.

Fig. 3.10 Overview of the European \& Developing Countries Clinical Trials Partnership

euros from European countries and Horizon 2020. The ENDORSE project, described below, is one of the project in the second EDCTP programme.

- Enhancing individual and institutional infectious disease outbreaks response capacities of healthcare professionals to mitigate infections emergencies in the Northern Uganda region (ENDORSE). The project (2016-2017) provides training to healthcare workers in biosafety and protection, against infectious diseases, and focuses on the region of Northern Uganda, where capacity building will benefit healthcare workers in both laboratories and patient-care settings. The project addresses the existing gaps in human resources for health, the wide disparities in health status across the country and the weakness of capacity in planning, management and human resource development. To achieve these objectives, a sustainable Train-the-Trainer model will be implemented and tested through training phases. Uganda is the only participant from Sub-Saharan Africa, joining European participants from Italy and Ireland. ENDORSE is funded with almost 200,000 euros by EDCTP.

\section{The African Union Research Grants Programme}

The AURG was initiated by the Department of Human Resources, Science and Technology (DHRST) of the AUC to support a pan-African R\&D programme through grants and direct funding. In line with the African 
Start date: 2014 - End date: 2020

Total budget: 17.5 million euros allocated in the second phase

Funding body: European Union

In brief: The AURG programme supports Pan African research and development through grants and direct funding, as tools for sustainable development, as well as building and strengthening Africa's SET capacities.

Fig. 3.11 Overview of the African Union Research Grants programme

Union's Science Technology and Innovation Strategy (STISA-2024) and with the JAES and the Priority 3 on human development of the EU-Africa partnership, the AURG programme supports collaborative research and R\&I activities contributing to the sustainable development of African countries. A key priority is to develop the capacity of the AUC to design, implement and monitor R\&I funding programmes, to establish the basis for a credible and reputable African framework programme for R\&I (to attract additional funds from other sources such as AU member states and other partners and donors), and to enhance intra-regional and NorthSouth scientific research consortia (Fig. 3.11).

Already in its second phase, the AURG programme is financed through a financial agreement between the EC and the AUC under the pan-African programme (2014-2020), whereby the EC has allocated a budget of 17.5 million euros for two calls in 2016 and 2017. The call launched in 2016 awarded grants to research projects addressing the priorities set out in the R\&I Roadmap on Food \& Nutrition Security and Sustainable Agriculture, namely eradicating hunger and ensuring food and nutrition security, which were determined through the EU-Africa High Level Policy Dialogue on STI.

\section{Africa-Europe STI Cooperation Programming:}

\section{A LOOK FORWARD}

The thematic areas in which Africa-Europe cooperation on STI takes place have expanded both qualitatively and quantitatively over the years. Furthermore, the link between research areas and priorities, funding instruments, and joint strategies increasingly reflect common values and 
objectives as opposed to the EU's one-sided agenda setting of the past. Nevertheless, this dynamic and rich landscape is not without challenges: the private sector is still under-represented and Africa-wide participation in bi-regional programming is still uneven.

Although most of the research funding and programming described above are open for private sector participation, its involvement remains minimal, and African participation in cooperation projects remains limited to a few public universities and research organisations. The long-term impact of applied research activities may be limited, if they are not coordinated with commercial actors who are interested to develop new processes, products and services. Low levels of private sector participation can be explained by a limited level of awareness among private sector actors regarding opportunities under FP7 and Horizon 2020; the subsequent lack of effective response to the calls proposals; and the lack of strategic alliances with European institutions and consortia.

Outcome Testimonial: Strengthening African capacities in collaborative relations with the EU within STI. Compiled by Jean Albergel, Mamobloding Tlhagale, Johan Viljoen (Institut de Recherche pour le Développement, IRD) and Toto Matshediso (South African Department of Science and Technology).

ER Africa was launched in early 2011 as the result of a number of European and African countries being eager to better coordinate and strengthen their individual bilateral collaborative relations in STI. The project was aimed at belping to realise the first action plan of the JAES, seeking in particular to strengthen African capacities in STI. The concept of such a project was expressed during a CAAST-Net stakeholders' meeting in Mombasa, Kenya, in November 2009. The consortium in charge of the ambitious ERAfrica project was mainly composed of partners from CAAST-Net.

Through ERAfrica, funding parties from 15 African and European countries jointly created the necessary funding mechanisms and processes leading up to a first call for research proposals in which partners participated on an equal footing. The joint call for projects covered three thematic fields: renewable energies, interfacing challenges and new ideas. Project could involve three types of collaborative activities including collaborative research, collaborative innovation and capacity building. With a total of 10.7 million euros available for funding, the call generated 124 proposals from which ERAfrica selected 17 projects to be funded. The selected projects represent a total amount of 8.29 million euros and 65 institutions (31 in Africa) from 18 countries (8 African countries), working together in these projects. 
As 9 African institutions and 8 European institutions have the important role of project coordination, the overall picture shows that ERAfrica indeed lives up to its aim of "true" partnerships and enhanced African capacities within research collaboration. These ERAfrica-funded projects are good examples of how research, development and innovation can be used to improve the lives of the African and European citizens and particularly in the field of bealth systems, food and nutritional security. At the end of the first phase of ER Africa, there was a great desire among the funding parties to see it continue even if it would be without funding from the EC.

A number of instruments exist to enhance and facilitate international collaboration and promote the internationalisation of non-European SMEs. If properly and more widely implemented, they could bring significant improvements to international cooperation in R\&I. The Business Cooperation Centres (BCCs), established through the initiative of the Enterprise Europe Network (EEN) in major international growth markets (such as Brazil, Russia, China and India) is one of them. The BCCs serve as a contact point for EU SMEs to enter international markets and to establish connections with local firms. In Sub-Saharan Africa, Cameroon and Nigeria have already established BCCs in order to facilitate business, technology as well as research partnerships between local SMEs and European SMEs based on common interests and a desire for mutual benefits (EEN 2016). More recently, the Enterprises of Cameroon were established in Douala. They are a professional association with expertise on how to facilitate cross-border commercial cooperation. Likewise, Nigeria established the Nigerian-Belgian Chamber of Commerce in Lagos, with the objective of promoting collaboration between Nigerian and Belgian enterprises by creating a platform and a friendly environment to support business development. BCCs can provide an effective platform for SMEs to internationalise their business and to explore R\&I partnerships.

The NCPs network is another tool created to improve the implementation of the funding instruments of the EU. The EC considers NCPs to be vital partners for the implementation of funding programmes such as Horizon 2020. An NCP is a trained individual, officially appointed by its host organisation (upon EC endorsement) whose mission is to guide his/her country's participation in EU-funded 
programmes. NCPs serve as a source of strategic information. They advise SMEs and other organisations on how to access European consortia, through acquiring knowledge of the latest developments in R\&I and gaining access to a pool of international partners for future collaboration. Furthermore, NCPs provide free of charge support in the applicant's own language.

Countries with well-established NCPs usually increase their participation in European programmes, through raised awareness of programmes, specific calls and technical requirements. Additionally, NCP networks help to identify pockets of excellence in the given country, ensuring their alignment with specific calls. In the case of Sub-Saharan Africa, there is evidence that the NCP network has been effective in enhancing participation in the calls for proposals in FP7. Indeed, the evolution of NCPs seems to be positively related to the country's increased participation in FP7 projects. $^{2}$ It is worth noting that other African countries have also increased their participation without an established NCP network, which can be explained by effective informal networks of researchers collectively applying to EU funding.

\section{Conclusion}

Although African countries have successfully participated in several EU funding programmes, African participation within Horizon 2020 appears to have decreased in comparison to FP7. At the same time, we see a wider range African countries applying to other funding schemes. It is evident that more work needs to be done to engage the private sector. This process must begin with unravelling the multiple limitations and barriers that private sector organisations experience in becoming aware of funding programmes, and how to access them. Experience has shown that there is limited understanding of the approaches and tools for enhancing innovation cooperation. Some existing tools have proven to be effective, and it would be useful to build on their experience. Networks such as the EEN can provide a great platform to strengthen the collaboration among companies and research organisations between Europe and Africa.

Bi-regional STI collaboration is increasingly becoming more reflective of the political aspiration towards co-ownership and equal partnership for 
mutual interest and mutual benefit. Focusing on areas of common interest and sharing common values are key ingredients of co-owning projects, and these are areas where significant improvements have been achieved. However, more remains to be done in terms of co-financing. The review of funding programmes in this chapter points out that bi-regional cooperation remains largely dependent on European funding. There has been some experience and experimentation with co-financing models showing excellent results, as exemplified by the ERAfrica consortium (under EU FP7), and the EDCTP. The recently established AURG programme, although still dependent on EU funding, is gradually building African institutional capacity to manage pan-African research programmes. Such programmes, eventually fully funded and owned by African countries, would expand the space for cooperation with international partners, including the EU, and would provide a richer and more diverse basis for bi-regional cooperation.

\section{Notes}

1. It must be noted that FP7, which spanned the period 2007-2013, had a longer duration than the FP6 (2002-2006). This may account for a proportion of the difference in total participation by and total financial allocation to African participants.

2. The efforts of CAAST-Net and CAAST-Net Plus have increased the NCP numbers in African countries. Through organising workshops and information days both on NCPs and Horizon 2020 the partners of CAAST-Net Plus have reached several countries in the Sub-Saharan region including Sudan, Cameroon, Angola, Mozambique, Mauritius, Uganda, Malawi, Tanzania, Kenya, Nigeria and Ghana (CAAST-Net 2013).

\section{REFERENCES}

CAAST-Net. (2009). Africa-Europe cooperation in science and technology: Status and way forward 10-11 November 2009 Mombasa, Kenya (summary report and recommendations). Available from: https://caast-net-plus.org/object/document/1285/attach/CAAST-Net_2009_Stakeholders__Conference_ Conclusions.pdf. Accessed 18 May 2016.

CAAST-Net Plus. (2013). Status of African national contact points. Available from: https://caast-net-plus.org/object/document/642/attach/D5_2_4_1_ African_NCP_status_report.pdf. Accessed 22 July 2016. 
COST. (2017a). A new COST success story: Green chemistry and cooperation with African countries. Available from: http://www.cost.eu/media/newsroom/ node_751. Accessed 27 June 2017.

COST. (2017b). CMST COST action D29 sustainable/green chemistry and chemical technology. Available from: http://www.cost.eu/COST_Actions/cmst/ D29. Accessed 27 June 2017.

EDCTP. (2014). Assessment of the performance and impact of the first programme of the European of developing countries clinical trials partnership (EDCTP). Available from: http://www.edctp.org/web/app/uploads/2015/03/Assessment-of-theperformance-and-impact-of-the-first-EDCTP-Programme_TechnopolisGroup_18SEP2014.pdf. Accessed 27 June 2017.

Engida, T., Nigist, A., Licence, P., \& Poliakoff, M. (2007). Empowering green chemists in Ethiopia. Available from http://science.sciencemag.org/content/316/5833/1849.full. Accessed 27 June 2017.

Enterprise Europe Network. (2016). A network outpost in sub-Saharan Africa. Available from: http://een.ec.europa.eu/news/news/network-outpost-subsaharan-africa Accessed 18 May 2016.

EUREKA. (2017). About Eureka. Available from: http://www.eurekanetwork. org/about-eureka. Accessed 27 June 2017.

European Commission. (2009). International cooperation with Africa in FP6. Brussels: European Commission. 
Open Access This chapter is licensed under the terms of the Creative Commons Attribution 4.0 International License (http://creativecommons.org/licenses/ by $/ 4.0 /$ ), which permits use, sharing, adaptation, distribution and reproduction in any medium or format, as long as you give appropriate credit to the original author(s) and the source, provide a link to the Creative Commons license and indicate if changes were made.

The images or other third party material in this chapter are included in the chapter's Creative Commons license, unless indicated otherwise in a credit line to the material. If material is not included in the chapter's Creative Commons license and your intended use is not permitted by statutory regulation or exceeds the permitted use, you will need to obtain permission directly from the copyright holder.

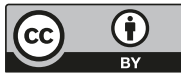

